Mareike Thies*, Jennifer Maier, Marc Levenston, Garry Gold, Rebecca Fahrig, Björn Eskofier, and Andreas Maier

\title{
Automatic Orientation Estimation of Inertial Sensors in C-Arm CT Projections
}

https://doi.org/10.1515/cdbme-2019-0050

\begin{abstract}
To obtain CT images of the knee joint in a more lifelike position, data acquisition can be performed with patients in standing rather than in lying position. However, in that situation, people tend to show involuntary motion. One possibility to compensate for that motion is the use of Inertial Measurement Units, that capture the accelerations during the scan. For this purpose, their local coordinate system needs to be known. An estimation based on the SIFT algorithm was implemented and compared to an existing approach that uses the Fast Radial Symmetry transform and to expert labels for evaluation. The SIFT method showed to be superior to the existing approach as it could extract stable feature points from the projections that were used to estimate the three-dimensional coordinate system in a reliable manner. The final algorithm achieved a mean euclidean distance of $2.61 \mathrm{~mm}$ between the calculated position of the origin and the assumed ground truth by the expert labels.
\end{abstract}

Keywords: Computed Tomography, Feature Extraction, Motion Compensated Reconstruction, SIFT

\section{Introduction}

Cone-beam C-arm CT systems enable the scanning of patients in standing weight-bearing position, thereby giving useful insights into the knee joint for monitoring the progression of Osteoarthritis. However, involuntary balancing of the patient during such acquisitions results in a considerable decrease in image quality of the reconstructed volumes. In order to obtain data usable for diagnosis in clinical applications, the patient's motion has to be estimated for the reconstruction.

Existing approaches for patient motion estimation propose to perform e.g. an image-based registration to a motionfree data set acquired in supine position [1], or to use external cameras that capture the surface of the knee during the scan

\footnotetext{
*Corresponding author: Mareike Thies,

Friedrich-Alexander-Universität Erlangen-Nürnberg, Erlangen, Germany, mareike.thies@fau.de

Jennifer Maier, Björn Eskofier, Andreas Maier,

Friedrich-Alexander-Universität Erlangen-Nürnberg, Erlangen, Germany

Marc Levenston, Garry Gold, Stanford University, Stanford, USA

Rebecca Fahrig, Siemens Healthcare GmbH, Erlangen, Germany
}

[2]. A well-working technique is to attach fiducial markers to the knee, that can be tracked in the projection images [3, 4], but the attachment is tedious and time-consuming. Hence, Maier et al. proposed to use Inertial Measurement Units (IMUs) for the purpose of motion estimation, where one single IMU attached to the knee is able to record the knee's motion [8].

For this approach, the IMU's local coordinate system needs to be known. In previous work, its estimation relied on the Fast Radial Symmetry Transform and required manual input [8]. Here, a method for automatically estimating the IMU's origin and axes is proposed that extracts features of the sensor containing the IMU in the CT projection images. The choice of a suited feature extractor fulfilling certain requirements is crucial. It needs to be invariant to orientation, overall intensity and size of the sensor, and robust against a change of viewpoint. The Hough transform for straight lines could be applied in order to find the sensor's housing contour [6], but since this has low contrast in the projections, a feature extraction based on the sensor's metal components was preferred. Localized features could also be extracted by Harris corner detector [5]. However, this method is sensitive to the image scale because it investigates variation in a fixed sized window. The Scale Invariant Feature Transform (SIFT) used in this work overcomes this scale problem as it operates on scale space pyramids. Furthermore, it is invariant to rotations and to some degree to viewpoint changes [12]. Another gain is that the found features are described with a unique vector, yielding a suitable feature extractor and feature descriptor that can be used for matching features in subsequent images [7].

\section{Materials and methods}

\subsection{CT Projection Data}

CT data was acquired under an IRB-approved protocol, containing four scans of a pumpkin phantom with motion applied, and five scans of a healthy subject's knees in a weight-bearing position. The C-arm (Artis Zeego, Siemens Healthcare GmbH, Erlangen, Germany) rotated on a horizontal trajectory acquiring 248 projections with a size of $1240 \times 960$ pixels and an isotropic pixel resolution of $0.308 \mathrm{~mm} /$ pixel. Figure 1 shows example projections of the phantom and the human scan. 


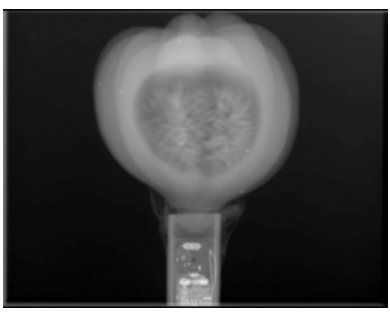

(a)

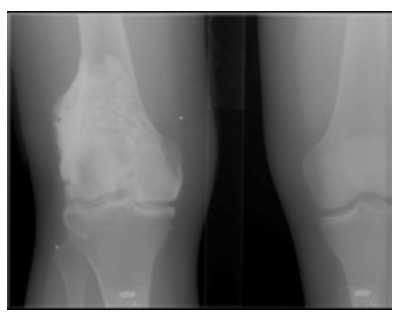

(b)
Fig. 1: Exemplary projection images of (a) the phantom data set, and (b) the human data set.

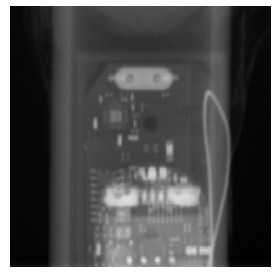

(a)

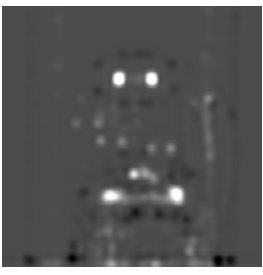

(b)

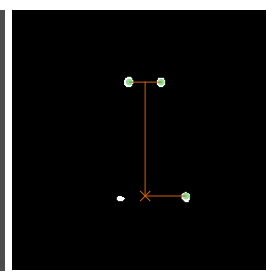

(c)
Fig. 2: Steps of reference approach for IMU tracking. (a) Original projection, (b) FRST transformed image, (c) binarized image with tracked points and estimated origin marked with cross.

\subsection{Semi-automatic reference approach}

To estimate the IMU's coordinate system, in [8], a semiautomatic tracking was performed. First, a Fast Radial Symmetry Transform was applied to the projections to find circular structures. The transformed images were then binarized, see Figure 2. In one binary projection image, the positions of three characteristic points on the sensor had to be marked manually. For each following projection, non-zero pixels surrounding the previous position were found by region growing, with the center of this region defining the new position. The 3D positions were determined by backprojecting the tracked points using the calibrated projection matrices.

Since the three tracked points were lying in one plane on the circuit board of the sensor, they could be used to define the IMU origin as depicted in Fig. 2(c).

\subsection{SIFT Algorithm}

The SIFT algorithm as presented by Lowe in 1999 [7] consists of three principal steps: Identification of key points in the image, assignment of a predominant orientation and calculation of a unique feature vector. The first step is based on the Difference of Gaussians (DoG) operator. It approximates the Laplacian by subtracting two versions of the image differing in the amount of Gaussian smoothing in order to detect corners or edges. Additionally, a scale space is built up by down-

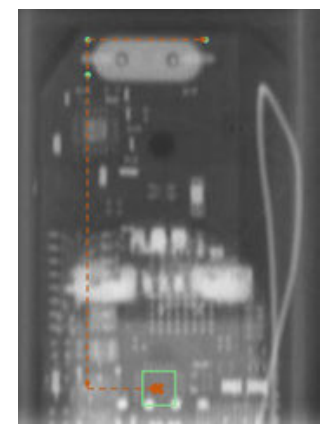

Fig. 3: Reference image showing the sensor, the position of the interest points (green) and of the IMU (green rectangle) having its origin in the center (red cross)

sampling the image after some steps of smoothing. The DoG is applied for every sampling rate making the key point detection independent of image scale. To achieve rotation invariance, each feature point is assigned an orientation based on the predominant direction of the gradient in a local neighborhood. Finally, a unique descriptor for each feature point is calculated by selecting a neighborhood around the key point, stretching the intensities inside this area to the interval $[0,1]$ to make the descriptor invariant to contrast changes [12] and computing the gradient for each pixel in the neighborhood. The final feature vector consists of the heights in a gradient histogram and serves as a unique description of the respective key point [9].

In this work, an implementation of the SIFT algorithm by Saalfeld and Tomančák [12] constructed as plug-in for the open source software ImageJ [11] was applied. It additionally applies a feature matching between two images based on the assumption that true matches have a much lower euclidean distance in the feature space than false pairs of key points.

\subsection{Final Pipeline}

A reference image of the sensor was extracted from one phantom data set, and the SIFT algorithm was applied once in order to detect the points producing the highest number of matches. These interest points are depicted in green in figure 3, where the green rectangle marks the IMU with its origin in the center.

The final implementation had the following basic steps (see figure 4): The SIFT algorithm is applied to every projection image with the extracted reference image. All SIFT parameters except the maximum image size were left to the values originally proposed by Lowe [7]. The feature point candidates belonging to the interest points are identified and their coordinates are saved. A 3D position is estimated from the identified 2D coordinates of the interest points using the system geometry. As most of the used data sets are corrupted by motion, this does not resolve to one point in the $3 \mathrm{D}$ volume. 


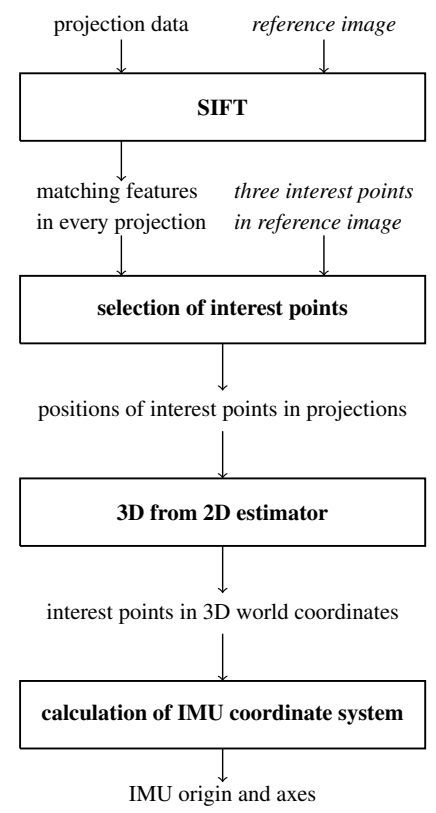

Fig. 4: Principal steps of the final feature extraction and coordinate system estimation

Therefore, a linear least squares approximation is used. Based on the dimensions of the sensor, origin and axes of the IMU's coordinate system can be calculated from the computed world points as shown in figure 3. All three interest points were assumed to be located in the plane of the circuit board holding the IMU and the vector connecting the two leftmost points was assumed to be parallel to the sensor's housing.

In the human scans, sensors are attached to both knees and visible in the projections. To make sure that all found matches refer to the same sensor, one has to be excluded prior to the SIFT algorithm. The knee of interest and its attached sensor were determined based on the bright contrast agent that was injected before scanning. Projections in the lateral view with overlapping knees and sensors had to be excluded manually.

\section{Results}

The coordinate systems defined by the proposed approach were compared to those of the reference algorithm. Additionally, the estimated origins were evaluated using expert labels.

Table 1 contains the euclidean distances of the origins computed by the SIFT algorithm to the reference approach origins, and to the expert labels for the phantom data sets. The origins of the proposed algorithm and the expert labels differ by a mean of $2.61 \mathrm{~mm}$ with a maximum of $4.62 \mathrm{~mm}$, whereas the mean distance of reference approach to expert labels is larger with $11.79 \mathrm{~mm}$ and little variation over the different data sets.

\begin{tabular}{cccc}
\hline \multirow{2}{*}{ Data set } & \multicolumn{3}{c}{ Distance [mm] between } \\
& SIFT and ref & SIFT and expert & ref and expert \\
\hline 1 & 12.01 & 1.85 & 12.71 \\
2 & 12.00 & 1.20 & 12.19 \\
3 & 11.04 & 2.76 & 11.35 \\
4 & 11.92 & 4.62 & 10.90 \\
\hline
\end{tabular}

Tab. 1: Euclidean distances between the origins of the proposed approach (SIFT), the reference approach (ref) and the expert labels on the phantom data sets.

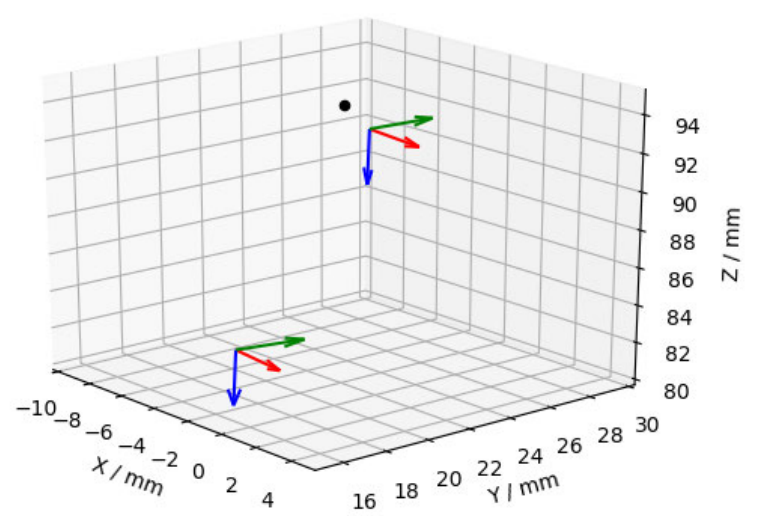

Fig. 5: Coordinate systems as found by reference approach (lower left) and by proposed SIFT method (upper right), the origin as labelled by the expert is shown as black point

Figure 5 shows the coordinate systems estimated by the SIFT method and the reference approach as well as the expert origin label for an exemplary data set. The offset between the respective origins is visible, and the proposed approach is closer to the expert labelled origin than the reference approach. The orientation of the axes is nearly identical. This observation also holds for the other data sets. The euclidean distance between the axes of the SIFT method and the reference approach is $0.11 \pm 0.08 \mathrm{~mm}$.

Since the IMU is not visible in the human projection data, the qualitative evaluation is based on the interest points in this case. As shown in figure 6, the interest points have approximately the expected positions. However, they show a higher variation compared to the phantom data.

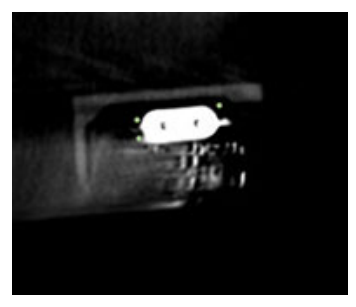

Fig. 6: A slice of the reconstruction of a human scan showing the visible part of the sensor and the found interest points (green). 


\section{Discussion}

The proposed SIFT algorithm successfully estimated the IMU coordinate system in a reliable manner for the phantom scans. The estimated axes agree very well with those found by the reference approach. Moreover, a small euclidean distance of calculated origin to expert labelled origin was achieved. The differences to the reference approach are larger but show a low variation over the data sets. This indicates a systematical deviation that is probably the consequence of different assumptions about the correct position of the origin, or of slightly imprecise sensor measurements impacting the calculation.

The application of the SIFT approach on the human data sets reveals some of its problems. It cannot be applied to images containing two sensors, consequently such images need a workaround. Since the three interest points have a small distance from each other small uncertainties in their calculated 3D position will have a severe impact on the estimated origin. An improvement could be to choose interest points spread over a larger area of the sensor or to use more than three interest points for the calculation. In this particular case, this was not possible as just the upper part of the sensor was visible in the projection images of the human scans. This underlines the importance of choosing interest points in the reference image, because the method can only work if the positions of all interest points can be determined. Furthermore, the approach works best if the sensor in the reference image stored in advance has similar intensities and contrast as in the projections.

\section{Conclusion}

In this work, an approach for estimating an IMU's coordinate system based on SIFT features extracted from CT projection images was proposed, and compared to an existing reference approach and to expert labels. On the phantom data sets, the proposed approach showed to be superior to the reference approach, since the estimation worked without direct manual input and achieved higher accuracy. The human data sets caused some problems, especially because the sensor was not fully visible in the projections. Hence, the performance could be further improved by making sure that the sensor is completely covered in future scans. Additionally, markers could be attached directly to the sensor before scanning. Placed at positions well aligned with the coordinate axes and sufficiently far apart, they are potentially easier to track in the projections than the sensor components. The proposed approach could still be applied, but with adapted interest points, whose influence could be investigated in future studies.

\section{Author Statement}

This work was supported by the Research Training Group 1773 Heterogeneous Image Systems by the German Research Foundation (DFG). Further, the authors acknowledge funding support from NIH 5R01AR065248-03 and NIH Shared Instrument Grant No. S10 RR026714. Bjoern Eskofier acknowledges the support of the DFG within the framework of the Heisenberg professorship programmme (grant number ES 434/8-1). Authors state no conflict of interest. Informed consent has been obtained from all individuals included in this study. The protocol for X-ray C-arm CT acquisition was IRB approved.

\section{References}

[1] Berger, M, Müller K, Aichert A, Unberath M, Thies J, Choi $\mathrm{JH}$, et al. Marker-free motion correction in weightbearing cone-beam CT of the knee joint. Medical physics 2016;43:1235-1248.

[2] Bier B, Ravikumar N, Unberath M, Levenston M, Gold G, Fahrig $\mathrm{R}$, et al. Range imaging for motion compensation in $\mathrm{C}$-arm cone-beam CT of knees under weight-bearing conditions. Journal of Imaging 2018;4:13.

[3] Choi JH, Fahrig R, Keil A, Besier TF, Pal S, McWalter EJ, et al. Fiducial marker-based correction for involuntary motion in weight-bearing C-arm CT scanning of knees. Part I. Numerical model-based optimization. Medical physics 2013;40.

[4] Choi JH, Maier A, Keil A, Pal S, McWalter EJ, Beaupré GS, et al. Fiducial marker-based correction for involuntary motion in weight-bearing C-arm CT scanning of knees. II. Experiment. Medical physics 2014; 41.

[5] Harris CG, Stephens M. A combined corner and edge detector. Alvey vision conference 1988;15:147-151.

[6] Hough, PV. Method and Means for recognizing complex patterns. In: U.S. Patent No. 3069654. Washington, DC: U.S. Patent and Trademark Office; 1962.

[7] Lowe DG. Object recognition from local scale-invariant features. ICCV 1999;2:1150-1157.

[8] Maier J, Aichert A, Mehringer W, Bier B, Eskofier B, Levenston $\mathrm{M}$, et al. Feasibility of Motion Compensation using Inertial Measurement in C-arm CT. IEEE Nuclear Science Symposium and Medical Imaging Conference 2018.

[9] Nischwitz A, Fischer M, Haberäcker P, Socher G. Computergrafik und Bildverarbeitung Band 2: Bildverarbeitung. 3rd ed. Wiesbaden: Vieweg \& Teubner; 2011.

[10] Nixon M, Alberto SA. Feature extraction and image processing for computer vision. 3rd ed. Oxford, London: Academic Press; 2012.

[11] Rueden CT, Schindelin J, Hiner MC, DeZonia BE, Walter AE, Arena ET, et al. ImageJ2: ImageJ for the next generation of scientific image data. BMC bioinformatics 2017;18(1):529.

[12] Saalfeld S, Tomancák P. Automatic landmark correspondence detection for ImageJ. Proceedings of the ImageJ User and Developer Conference 2008;128-133. 